\title{
Medium modifications of the nucleon-deuteron break-up cross section in the Faddeev approach
}

\author{
M. Beyer, G. Röpke and A. Sedrakian* \\ Max-Planck-Gesellschaft, AG 'Theoretische Vielteilchenphysik' \\ Universität Rostock, Universitätsplatz 1, 18055 Rostock, Germany
}

\begin{abstract}
The three-nucleon scattering problem in a nuclear medium is considered within the Faddeev technique. In particular the deuteron break-up cross section that governs the formation and the break-up reactions of deuterons $(N N N \leftrightarrow N d)$ in a nuclear environment is calculated at finite temperatures and densities. A significant enhancement of the in-medium break-up cross section with increasing density has been found.
\end{abstract}

Formation of light clusters such as deuterons, helium and alpha particles is an important aspect of heavy ion collisions at intermediate energies, see e.g. [1]. Empirical evidence, including recent experimental data on cluster formation, see refs. [2.3], indicate that a large fraction of deuterons can be formed in heavy-ion collision of energies $E / A \leq 200 \mathrm{MeV}$.

During the expansion of the system the density can drop below the Mott-density of deuteron dissociation [4 6 . In this region the deuteron abundances will be determined by deuteron formation, $N N N \rightarrow d N$, and break-up, $d N \rightarrow N N N$, reactions. Since the deuteron formation rate can be expressed through the break-up probability, the final outcome of the reaction will be essentially controlled by the deuteron break-up cross section. Previous studies of the kinetics of deuteron production have utilized the impulse approximation to calculate the reaction cross section at energies above $200 \mathrm{MeV} / \mathrm{A} \mathrm{[7]}$. For lower energies, viz. $E / A \leq 200 \mathrm{MeV}$, the impulse approximation fails and a full three-body treatment of the scattering problem is necessary. Furthermore, a consistent treatment of cluster formation in expanding hot and dense matter requires the inclusion of medium effects into the respective reaction cross sections.

The essentials of the three-body problem in the vacuum case are well known, see e.g. ref. [8]. In the following we utilize the AGS formalism [9] suitably modified, in order to treat the three-body problem taking into account the nuclear medium in the quasi-particle approximation. To this end, we will rely on a separable representation of the nucleon-nucleon potential. This choice simplifies the problem considerably. A systematic investigation of separable parameterizations of "realistic" potentials has been pursued e.g. by Plessas and collaborators [10]. We note that solutions of the three-body problem using "realistic" $N N$ potentials have been achieved e.g. by the Bochum group [11], and the Bonn group in the framework of the $W$-matrix approach [12].

Recently, separable approximations have been used to solve the pion deuteron scattering problem [13] and successfully applied to coherent photo- and electro-production of pions on the deuteron [14. A detailed discussion of the numerical method used in these cases has been given in [15]. In the present work we have modified this pion deuteron code [16] to solve the nucleon-deuteron scattering problem. Our results for the dominant three-body transition matrix elements agree within $\simeq 5 \%$ with the previous calculations by Doleschall [17].

The AGS equations for the transition matrix $U_{\alpha \beta}(z)$ are given in a compact notation by [9]

$$
U_{\beta \alpha}(z)=\left(1-\delta_{\alpha \beta}\right) G_{0}^{-1}(z)+\sum_{\gamma=1}^{3}\left(1-\delta_{\alpha \gamma}\right) T_{\gamma}(z) G_{0}(z) U_{\gamma \alpha}(z),
$$

where the Greek indices represent particle channels. In the absence of the medium the free three-body resolvent is $G_{0}(z)=\left(z-H_{0}\right)^{-1}$ with $z=E+i \varepsilon$, where $H_{0}$ is the Hamiltonian of the noninteracting three-body system and $E$ is the three-body energy. The three-body transition matrix $T_{\gamma}(z)$ in the channel $\gamma$ is related to the $t$-matrix of the two-body subspace $\hat{T}_{\gamma}(\hat{z})$ (denoted by "hat") via,

$$
\left\langle\overline{\mathbf{q}}_{\gamma} \overline{\mathbf{p}}_{\gamma}\left|T_{\gamma}(z)\right| \mathbf{q}_{\gamma} \mathbf{p}_{\gamma}\right\rangle=\left\langle\overline{\mathbf{q}}_{\gamma} \mid \mathbf{q}_{\gamma}\right\rangle\left\langle\overline{\mathbf{p}}_{\gamma}\left|\hat{T}_{\gamma}\left(\hat{z}_{\gamma}\right)\right| \mathbf{p}_{\gamma}\right\rangle,
$$

*on leave of absence from Physics Department, Yerevan State University, Armenia 
with $\hat{z}_{\gamma}=z-3 q_{\gamma}^{2} /(4 m)$, where $m$ is the nucleon mass, and $\mathbf{p}_{\gamma}\left(\mathbf{q}_{\gamma}\right)$ are the three-body momenta in the center of mass frame for the pair (odd) nucleon, respectively. For a separable two-body potential, which is given by (overall particle channel index $\gamma$ suppressed)

$$
\hat{V}_{r}=\left|g_{r}\right\rangle \lambda_{r}\left\langle g_{r}\right|,
$$

where $r$ denotes a set of quantum numbers providing a complete specification of the two particle state, the two-body $t$-matrix is then given by

$$
\hat{T}_{r}(\hat{z})=\left|g_{r}\right\rangle \hat{\tau}_{r}(\hat{z})\left\langle g_{r}\right| .
$$

Solving the two particle Lippmann-Schwinger equation determines $\hat{\tau}_{r}$ (see e.g. [5])

$$
\hat{\tau}_{r}(\hat{z})=\left(\lambda_{r}^{-1}-\left\langle g_{r}\left|\hat{G}_{0}(\hat{z})\right| g_{r}\right\rangle\right)^{-1} .
$$

Multiplying the AGS equations with $\left\langle g_{\beta m}\right| G_{0}(z)\left[G_{0}(z)\left|g_{\alpha m}\right\rangle\right]$ from left [right] they reduce to [18]

$$
X_{\beta n, \alpha m}(z)=Z_{\beta n, \alpha m}(z)+\sum_{\gamma r} Z_{\beta n, \gamma r}(z) \tau_{\gamma r}(z) X_{\gamma r, \alpha m}(z)
$$

where

$$
\begin{aligned}
X_{\beta n, \alpha m} & =\left\langle g_{\beta n}\left|G_{0}(z) U_{\beta \alpha} G_{0}(z)\right| g_{\alpha m}\right\rangle, \\
Z_{\beta n, \alpha m} & =\left\langle g_{\beta n}\left|\left(1-\delta_{\beta \alpha}\right) G_{0}(z)\right| g_{\alpha m}\right\rangle .
\end{aligned}
$$

We restrict the two-body channels to the dominant ones, i.e. ${ }^{1} S_{0}$ and ${ }^{3} S_{1}-{ }^{3} D_{1}$. For the separable ansatz we use the parameterization of Phillips [19]. The parameters are taken from Ref. [20], which shows that gross features of the elastic and break-up cross sections as well as the differential elastic cross section up to $E_{l a b}=50 \mathrm{MeV}$ are sufficiently well reproduced. To calculate the break-up cross section we use the optical theorem. Figure 1 shows our results for the integrated cross section along with the experimental data [21].

Note that the agreement between the present calculations on free nucleon-deuteron scattering and the experimental data can still be improved, through using more realistic potentials and including contributions of higher partial waves. However, our present goal is to study the general features related to the inclusion of nuclear medium, which are expected to be quite significant, cf. [22].

The influence of the nuclear medium on the three particle problem is treated in mean-field approximation. Here, two effects appear, i) self energy corrections and ii) Pauli blocking. The single particle propagator is obtained within the Hartree-Fock approximation of the self energy. The Hartree-Fock energy shift is given by $(V(12,12) \equiv\langle 12|\hat{V}| 12\rangle)$

$$
\Delta^{H F}(1)=\sum_{2}[V(12,12)-V(12,21)] f(2),
$$

where the multi-indices 1,2 denote momentum and other quantum numbers of the respective particles, the Fermi function is given by $f\left(\varepsilon_{1}\right)=\left\{\exp \left[\left(\varepsilon_{1}-\mu\right) / T\right]+1\right\}^{-1}$, and the single particle energy by $\varepsilon_{1}=k_{1}^{2} / 2 m+\Delta^{H F}(1)$. The Hartree-Fock shift is evaluated using the same potential as for solving the AGS equations. To simplify the three-body treatment we introduce the effective mass approximation. The quasi-particle dispersion relation is then written as $\varepsilon_{1}=k_{1}^{2} / 2 m^{*}+\Delta_{0}^{H F}$, where the constant term $\Delta_{0}^{H F}$ can be absorbed into the chemical potential $\mu$. The effective mass $m^{*}$ is density and temperature dependent, and decreases from the value $m^{*} / m=1$ in the zero density limit to e.g. $m^{*} / m=0.97$ at $T=10 \mathrm{MeV}$ for the Mott density of the deuteron $n=8 \times 10^{-3} \mathrm{fm}^{-3}$.

The Bethe-Salpeter equation for the three-body Green's function $G(\overline{1} \overline{2} \overline{3}, 123 ; z)$ in mean field approximaton, including Pauli blocking and self energy shifts, is obtain from the Matsubara Green's function technique as

$$
\begin{aligned}
& G(\overline{1} \overline{2} \overline{3}, 123 ; z)=\frac{1-f(1)-f(2)-f(3)}{z-\varepsilon_{1}-\varepsilon_{2}-\varepsilon_{3}}\langle\overline{1} \overline{2} \overline{3} \mid 123\rangle \\
& +\sum_{1^{\prime} 2^{\prime} 3^{\prime}} G\left(\overline{1} \overline{2} \overline{3}, 1^{\prime} 2^{\prime} 3^{\prime} ; z\right)\left(V\left(1^{\prime} 2^{\prime}, 12\right)\left\langle 3^{\prime} \mid 3\right\rangle \frac{1-f(1)-f(2)}{z-\varepsilon_{1}-\varepsilon_{2}-\varepsilon_{3}}+\text { cycl. perm. }\right)
\end{aligned}
$$

This leads to modifications of the quantities entering in the AGS equations. Pauli blocking then appears in the transition matrix $T_{\gamma}(z)$ and the resolvent $G_{0}(z)$. The thermodynamic $t$-matrix acting in channel $\gamma$, see eq. (2)), has 
been derived within the Matsubara-Green's function techniques, see Ref. [23]. It turns out that, in the low density approximation used here, the effect of the Pauli blocking may be absorbed into an effective potential. Inclusion of all medium corrections effectively results in the following replacements in the eqs. (沟), (5), (6), and (8), viz.

$$
\begin{aligned}
m & \rightarrow m^{*}, \\
g_{\alpha m} & \rightarrow g_{\alpha m}^{*}=\left\langle Q_{\alpha}\right\rangle^{1 / 2} g_{\alpha m} .
\end{aligned}
$$

Here $\langle Q\rangle$ is the angular averaged Pauli blocking factor,

$$
\langle Q(\mathbf{p}, \mathbf{q})\rangle=2 \pi \int_{-1}^{1} d x\left[1-f\left(\varepsilon_{\mathbf{q} / 2+\mathbf{p}}\right)-f\left(\varepsilon_{\mathbf{q} / 2-\mathbf{p}}\right)\right] .
$$

where $x=\mathbf{p} \cdot \mathbf{q} /(p q)$. The center of mass of the three nucleon system is set equal to the momentum of the medium. The AGS equations are properly symmetrized for identical particles and then solved in the rest system of the medium.

We present our results with amplitudes normalized to the residue of the two-body $t$-matrix in the deuteron channel $d$, i.e.

$$
N_{\gamma d}^{* 2} \equiv \operatorname{Res}\left(\hat{\tau}, \hat{z}=E_{\gamma d}^{* B}\right)=\left\langle g_{\gamma d}^{*}\left|\hat{G}_{0}^{2}\left(E_{\gamma d}^{* B}\right)\right| g_{\gamma d}^{*}\right\rangle^{-1},
$$

where $E_{\gamma d}^{* B}$ is the in-medium deuteron binding energy. The advantage is that the optical theorem, which is used to calculate the break-up cross section, has the same formal structure as in the vacuum case and is valid without any further modifications. For an alternative formulation of the in-medium optical theorem in the two-body case see e.g. Ref. [5. The in-medium break up cross section $\sigma_{n, T}^{*}\left(E_{l a b}\right)$ that depends on density $n$ and temperature $T$ is then defined using the amplitudes $N_{\beta d}^{*} X_{\beta d, \alpha d}^{*}(z) N_{\alpha d}^{*}$ resulting from solving eq. (6) with the replacements given in eqs. (11) and (12) in the same way as in the vacuum case, see e.g. [8] or [13,24].

Our results are depicted in Figure 2. The solid lines represent the free space break-up cross section as shown in Figure 1. The dashed lines show the break-up cross section, $\sigma_{n, T}^{*}\left(E_{l a b}\right)$ for densities $n=0.1,1,3,5,7 \times 10^{-3} \mathrm{fm}^{-3}$, respectively, as a function of the laboratory energy $E_{l a b}$. The Mott transition occurs at the density $n=8 \times 10^{-3}$ $\mathrm{fm}^{-3}$.

The medium effects significantly modify the vacuum break-up cross section. Two qualitative features are observed. First, the break-up threshold is shifted towards lower scattering energies with increasing density of the nuclear matter. This kinematical effect is due to the decrease of the deuteron binding energy with increasing density. For an illustration of the dependence of the threshold energy in the laboratory system $E^{\text {th }}$ on different temperatures and densities see Figure 3. Second, the cross section increases considerably with increasing density. The maximum is enhanced by one order of magnitude for the largest density value considered. For densities larger than the Mott densities the deuteron disappears as a bound state.

Also, it is instructive to see how the medium dependent cross section converges to the "free" cross section. At 100 $\mathrm{MeV}$ the deviation of the in-medium cross section from the free in this model is in the order of $10 \%$. From inspection of Figure 2 we conclude that the dominant changes in the cross section takes place at rather moderate energies, i.e. where the impulse approximation fails, and the Faddeev technique has to be used.

To summarize, we have calculated the deuteron break-up cross section, $\sigma_{n, T}^{*}\left(E_{l a b}\right)$, using the AGS formalism modified in order to take into account the effects of quasi-particle energies and the phase space occupation effects. It has been shown that there are strong modifications of the deuteron break-up cross section compared to the vacuum scattering case. A study of the reactions dynamics within a kinetic approach to the cluster formation [25] 227], which will include the deuteron break-up and formation probabilities based on the present work, would provide an improved description of the physics of deuteron production in heavy-ion reactions.

We gratefully acknowledge B.L.G. Bakker for providing us with a three-body pion deuteron code. One of us (M.B.) would like to thank B.L.G. Bakker and F. Blaazer of VU Amsterdam for very fruitful discussions on numerical issues. G.R. and A.S. are thankful to V.B. Belyaev and W. Plessas for discussions.

[1] S. Nagamiya et al., Phys. Rev.C24 (1981) 971, and refs. therein.

[2] G. Kunde, Ph. D. thesis, GSI Darmstadt (1994), unpublished.

[3] Handzy et al. Phys. Rev. Lett. 75 (1995) 2916; Zhu et al., Phys. Rev. C 52 (1995) 784. 
[4] M. Baldo, U. Lombardo, and P. Schuck, Phys. Rev. C 52 (1995) 975.

[5] M. Schmidt, G. Röpke, and H. Schulz, Ann. Phys. (NY) 202 (1990) 57.

[6] T. Alm, G. Röpke, and M. Schmidt, Z. Phys. A 337 (1990) 355; G. Röpke, M. Schmidt, L. Münchow, and H. Schulz, Nucl. Phys. A 339 (1983) 587; T. Alm, L. Friman, G. Röpke, H. Schulz, ibid. A 551 (1993) 45; G. Röpke, Ann. Phys. (NY) 3 (1994) 145.

[7] P. Danielewicz and G.F. Bertsch, Nucl. Phys. A 533 (1991) 712.

[8] W. Glöckle, The Quantum Mechanical Few-Body Problem, Berlin-Heidelberg-New York (Springer) 1983; I.R. Afnan and A.W. Thomas in: Modern Three Hadron Physics ed. A.W. Thomas, p.1, Berlin-Heidelberg-New York (Springer) 1977; E.W. Schmidt and H. Ziegelmann, The Quantum Mechanical Three-Body Problem, Oxford (Pergamon Press) 1974; W. Sandhas, Acta Physica Austriaca, Suppl. IX, (1972) 57, V.B. Belyaev, Lectures on the Theory of Few-Body Systems (Spinger) Berlin 1990.

[9] E.O. Alt, P. Grassberger, and W. Sandhas, Nucl. Phys. B 2 (1967) 167

[10] W. Plessas et al., Few-Body Systems Suppl. 7, 251 (1994), and ref. therein.; L. Mathelitsch, W. Plessas, and W. Schweiger, Phys. Rev. C 26 (1982) 65; J. Haidenbauer and W. Plessas, Phys. Rev. C 30 (1984) 1822.

[11] H. Witala, T. Cornelius, and W. Glöckle, Few-Body 5 (1988) 89; J.L. Friar, G.L. Payne, W. Glöckle, D. Huber, and H. Witala, Phys. Rev C 51 (1995) 2356, and ref. therein.

[12] T. Januschke, T.N. Frank, W. Sandhas, and H. Haberzettl, Phys. Rev. C 47 (1993) 1401; T.N. Frank, H. Haberzettl, T. Januschke, U. Kerwath, and W. Sandhas, Phys. Rev. C 83 (1988) 1112, Th. Januschke, PhD. Thesis, Bonn 1990, BONN-IR-90-52, unpublished.

[13] A. J. Huizing, Ph.D. Thesis VU Amsterdam 1990.

[14] F. Blaazer, Ph.D. Thesis VU Amsterdam 1995.

[15] A.J. Huizing and B.L.G. Bakker, J. Comput. Phys. 90.1 (1990) 200.

[16] B.L.G. Bakker, private communication.

[17] P. Doleschall, Nucl. Phys. A 201 (1973) 264.

[18] C. Lovelace, Phys. Rev. 135 (1964) B1225.

[19] A.C. Phillips, Nucl. Phys. A 107 (1968) 209.

[20] J. Brunisma and R. van Wageningen, Nucl. Phys. A 282 (1977) 1.

[21] P. Schwarz et al., Nucl. Phys. A 398 (1983) 1.

[22] T. Alm et al., Nucl. Phys. A 587 (1995) 815.

[23] T. Alm, G. Röpke, and M. Schmidt, Phys. Rev. C 50 (1994) 31, and ref. therein.

[24] Y. Koike and Y. Taniguchi, Few-Body Systems 1 (1983) 13.

[25] M.B. Tsang, G.F. Bertsch, W.G. Lynch, and M. Tohyama, Phys. Rev. C 40 (1989) 1685.

[26] H.M. Xu, W.G. Lynch, P. Danielewicz and G.F. Bertsch, Phys. Rev. Lett. 65 (1990) 843.

[27] G. Röpke and H. Schulz, Nucl. Phys. A 477 (1988) 472. 


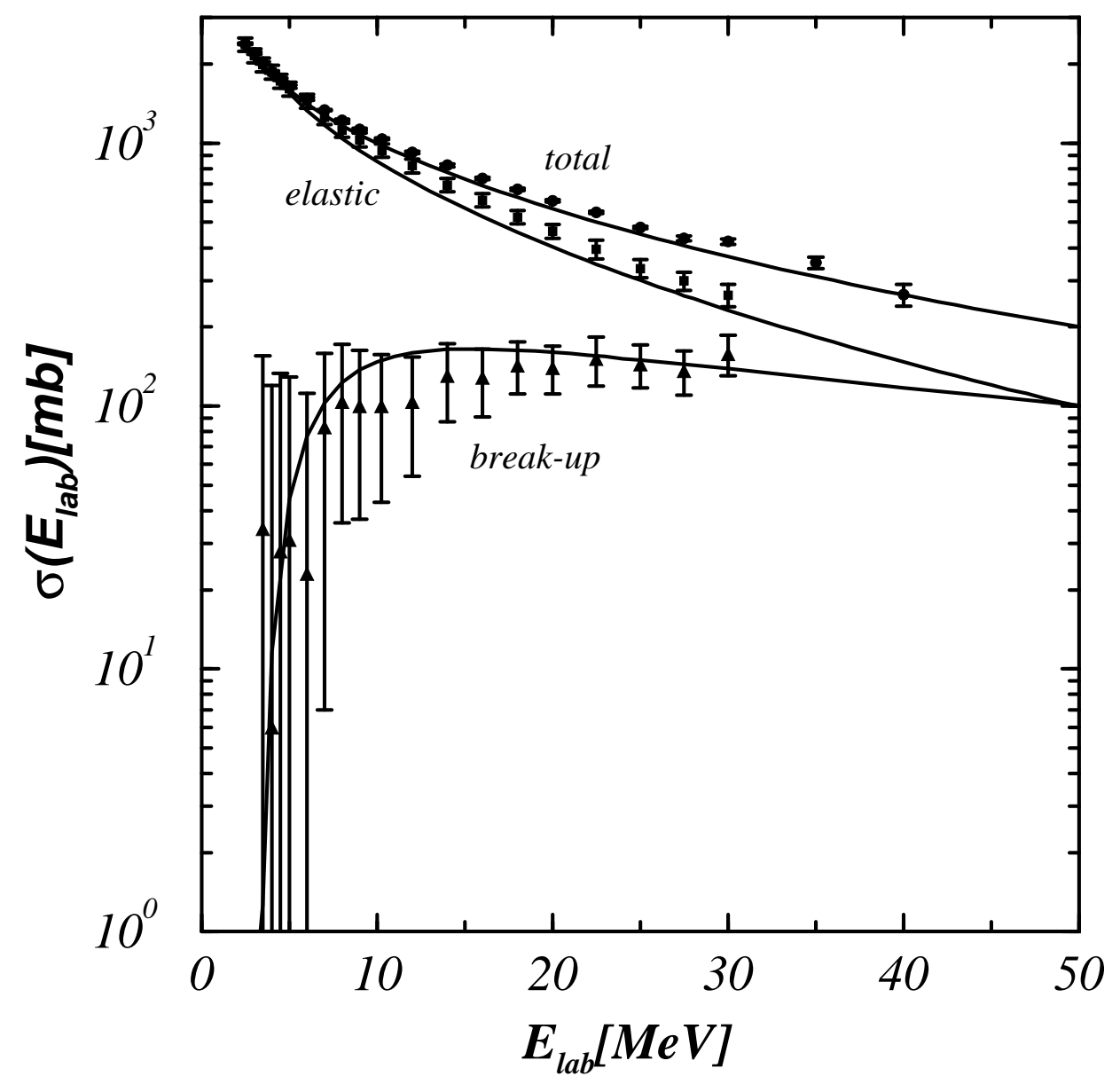

FIG. 1. A comparison of the total, elastic, and break-up cross sections with the experimental data of Ref. [21]. 


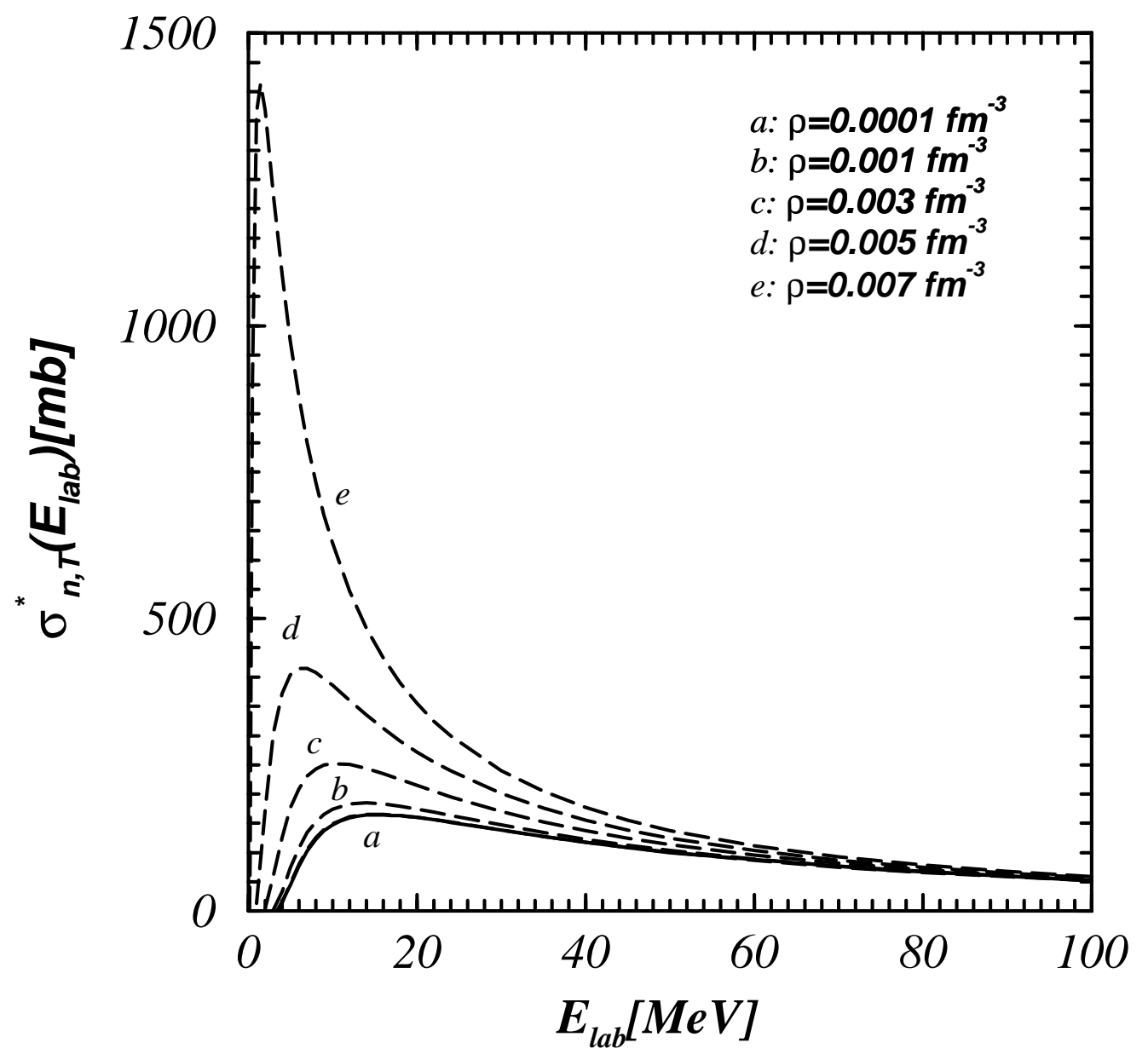

FIG. 2. Break-up cross section at temperature $T=10 \mathrm{MeV}$. Free cross section is shown as solid line. Other lines are due to different nuclear densities, see text. 


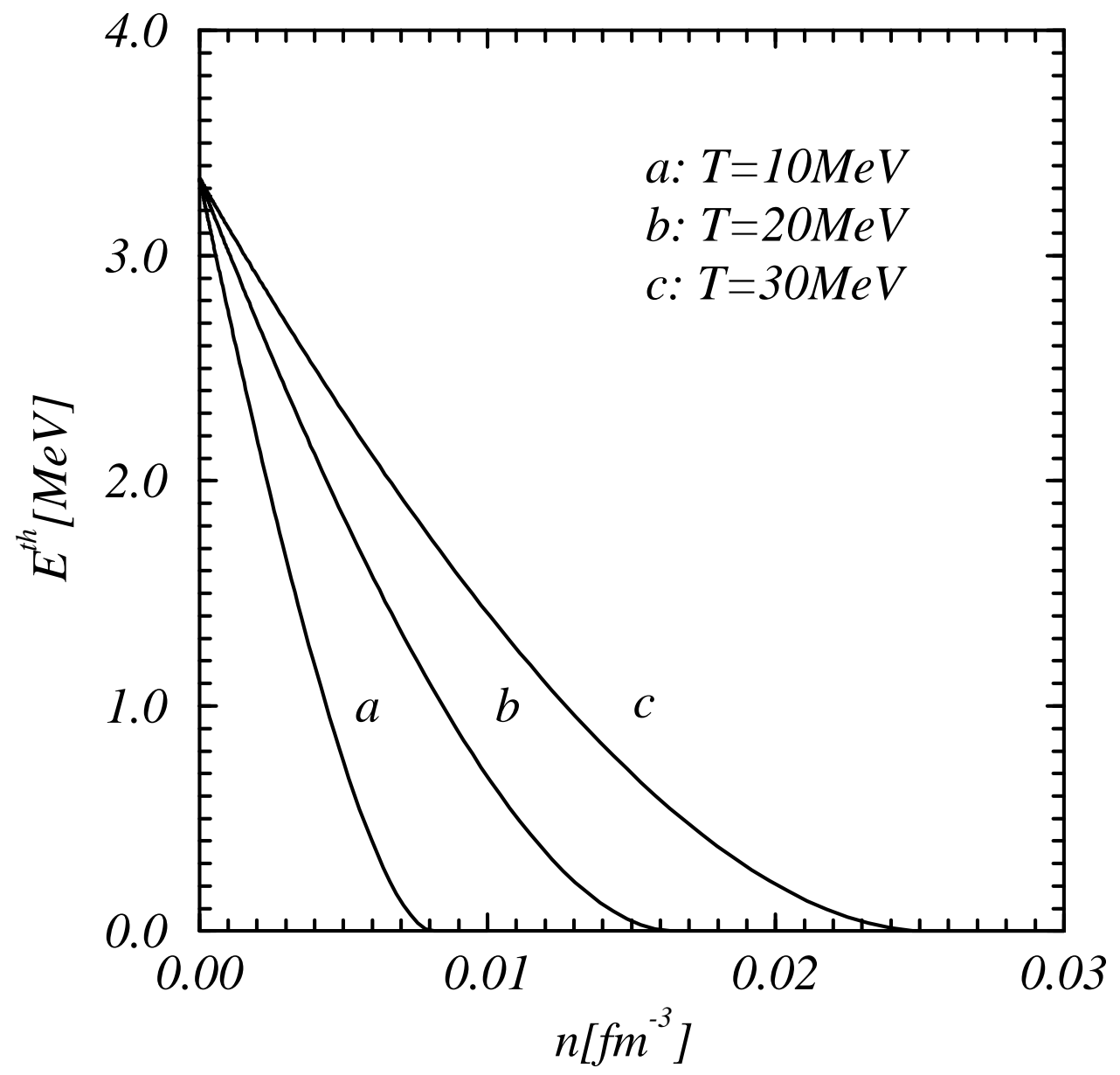

FIG. 3. Shift of threshold energy $E^{t h}$ in the laboratory system due to nuclear density $n$ at different temperatures $T$. 